\title{
Synthesis and Characterization of Poly(ether amide)s Containing Biphenylphthalazinone Moiety
}

\author{
Lianzhou CHEN and Xigao JiAN ${ }^{\dagger}$ \\ Department of Polymer Science \& Materials, Dalian University of Technology, \\ 116012, People's Republic of China
}

(Received December 4, 1998)

KEY WORDS Biphenylphthalazinone / Diacid / Diamine / Polyamides / Direct Polycondensation /

Linear aromatic polymers are known for their usefulness in meeting the high-performance requirements for structure resins, and polymer films, and coating materials need by the aerospace and electronics industry. Aromatic polymers, such as polyamides, polyimides, polyester, and poly(aryl ether ketone)s are the polymers of choice for these applications because of their unique combination of chemical, physical, and mechanical properties. Their low solubility and bad processing conditions limit the usage of these materials.

Aromatic polyamides constantly attract much interest because of their high-temperature resistance and excellent mechanical properties. ${ }^{1}$ In recent years interest has been focused on the synthesis of rigid-rod polyamides by incorporating aromatic heterocyclic rings into the polymer backbone. ${ }^{2-5}$ The increased number of aromatic heterocyclic rings enhance the rigidity of the polymer backbone, and polarizable $\pi$-electrons lead to strong intermolecular interactions increasing the transition temperatures of these polymers. Rigid-rod polymers also exhibit optical and morphological properties. ${ }^{6}$

Infusibility and limited solubility, are characteristic properties of rigid-rod polymers which restrict their synthesis, characterization, processing and applications, especially of high molecular weight materials. For example, poly ( $p$-phenylene terephthalamide) forms highstrength and high-modulus fibers $\left(\operatorname{Kevlar}^{\circledR}\right)$, but must be processed from concentrated sulfuric acid at elevated temperatures. ${ }^{7}$ Particularly, polyamides prepared from 4,4"-bis(chloroformyl)- $p$-terphenyl and 4,4"-diamino-p-terphenyl, benzidine, or 1,4-phenylenediamine are insoluble in polar aprotic solvents containing $\mathrm{LiCl}$, even in strong acids. ${ }^{8}$ Analogous behavior has been observed for poly(amide-imide)s prepared from 3,4-dicarboxy(4'-chloroformyl)biphenyl anhydride. ${ }^{9}$ Therefore, much effort has been made to increase the solubility of rigidrod polymers. ${ }^{10-14}$ These effort include: (1) introducing flexible segments into the polymer chains. (2) replacing symmetrical aromatic rings by the unsymmetrical ones, which lead to reduction in crystallinity. (3) incorporating bulky pendant groups along the polymer backbone to minimize crystallization. (4) forming a noncoplanar structure, thereby making crystallization impossible. (5) organometallic modification approach to increase solubility of aromatic polyamide. Rod-like polymers which are soluble in organic solvents without

\footnotetext{
$\uparrow$ To whom correspondence should be addressed.
}

the addition of inorganic salts enable not only processing routes such as dry spinning but also open applications in the area of films, coatings, polymer blends and composites.

Recently we prepared amorphous high-performance polymers containing 1,2-dihydro-4-phenyl- $2 \mathrm{H}$-phthalazinone moiety with very high glass transition temperatures and excellent thermooxidative stability, which are soluble in common organic solvents especially. ${ }^{15,16} \mathrm{On}$ basis of the above studies reported, we designed and synthesized a novel bisphenol 1,2-dihydro-4-(4-hydroxybiphenyl)-2H-phthalazine-1-one (1). From it, we prepared polymers with better properties than before. This paper extends our investigation to the synthesis of novel polyamides containing 1,2-dihydro-4-biphenyl-2H-phthalazinone moiety.

\section{EXPERIMENTAL}

\section{Monomer Synthesis}

1,2-Dihydro-4-(4-hydroxybiphenyl)-2 $\mathrm{H}$-phthalazine1-one (1): The synthesis of this bisphenol was according to the ref 17 , only using 4-hydroxylbiphenyl in place of $o$-phenylphenol. Yield: $78 \%$; mp $335^{\circ} \mathrm{C}$; IR $\left(\mathrm{cm}^{-1}\right)$ : 3280 , $3149,3020,1648,1607,1589,1462,1268,1209,1193$ (ms), 1175, 824, 799; ${ }^{1} \mathrm{H}$ NMR (ppm): 12.63 (s, 1H, NH), 9.31 (s, 1H, NH), 8.36 (m, 1H, 8-H); Anal. Calcd for $\mathrm{C}_{20} \mathrm{H}_{14} \mathrm{O}_{2} \mathrm{~N}_{2}: \mathrm{C}, 76.43 \% ; \mathrm{H}, 4.46 \% ; \mathrm{N}, 8.92 \%$; Found: C, 76.43\%; H, 4.63\%; N, 9.13\%.

1,2-Dihydro-2-(4-cyanophenyl)-4-(4-cyanophenyleneoxy)biphenyl-2,3-phthalazione-1-one (3): The condensation reaction was conducted in a flask equipped with a condenser, thermometer and nitrogen inlet. The reaction vessel was charged with $140 \mathrm{ml} N, N$-dimethylformamide (DMF), $80 \mathrm{ml}$ toluene, $0.05 \mathrm{~mol}$ 1,2-dihydro4-(4-hydroxybiphenyl)-2 $H$-phthalazine-1-one (1), 0.1 mol p-chlorobenzonitrile and $0.14 \mathrm{~mol}$ anhydrous potassium carbonate. The resulting solution was magnetically stirred and heated to reflux under nitrogen. The refluxing temperature was kept for $5 \mathrm{~h}$, and then the toluene was distilled. The reaction was kept $10 \mathrm{~h}$. The mixture was allowed to cool to room temperature and poured slowly into $250 \mathrm{ml}$ of water resulting in the formation of a water-insoluble solid, filtered to give the dinitrile compound. The product was recrystallized in water/ethanol to give white crystalline solid. Yield: $87 \%$; mp $224^{\circ} \mathrm{C}$; IR $\left(\mathrm{cm}^{-1}\right): 2227,1668,1650,1596,1495$, 1327, 1250, 1132, 843, 550, 415; ${ }^{1} \mathrm{H}$ NMR (ppm): 
$8.55-8.32(\mathrm{~m}, 1 \mathrm{H}), 7.73-8.05(\mathrm{~m}, 15 \mathrm{H}), 7.15-7.38(\mathrm{~m}$, $4 \mathrm{H})$; Anal. Calcd for $\mathrm{C}_{34} \mathrm{H}_{20} \mathrm{O}_{2} \mathrm{~N}_{4}$ : C, $79.07 \% ; \mathrm{H}, 3.87 \%$; N, 10.9\%; Found: C, 79.02\%; H, 4.04\%; N, 10.97\%.

1,2-Dihydro-2-(4-carboxylphenyl)-4-(4-carboxylphenyleneoxy)biphenyl-2,3-phthalazione-1-one (4): 4 was prepared using the following procedure: To a $250 \mathrm{ml}$ round bottom flask was added $8 \mathrm{~g}$ of $\mathrm{KOH}$ and $5 \mathrm{~g}$ of dinitrile compound in $50 \mathrm{ml}$ of ethanol and $75 \mathrm{ml}$ of distilled water. The mixture was stirred under refluxing temperature. After the suspended reaction solution turned into clear, the refluxing temperature was kept another $18 \mathrm{~h}$. The mixture was allowed to cool to room temperature and poured into diluted acid solution. The resulting solid was filtered, washed with water, and dried to give the yellow-white powder. Yield: $95 \%$; mp $303^{\circ} \mathrm{C}$; IR $\left(\mathrm{cm}^{-1}\right): 3563-3069,3030,1720,1650,1598,1495$, $1420,1326,1243,1165,1102,875,832,772,693 ;{ }^{1} \mathrm{H}$ NMR (ppm): $7.10-7.50(\mathrm{~m}, 6 \mathrm{H}), 7.62-8.02(\mathrm{~m}, 13 \mathrm{H})$,

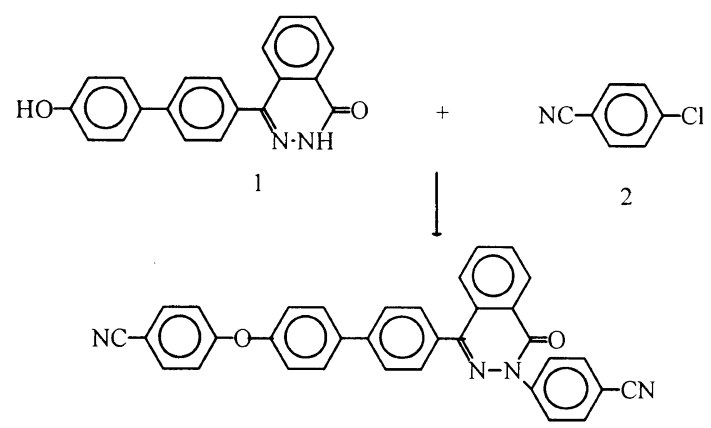

3

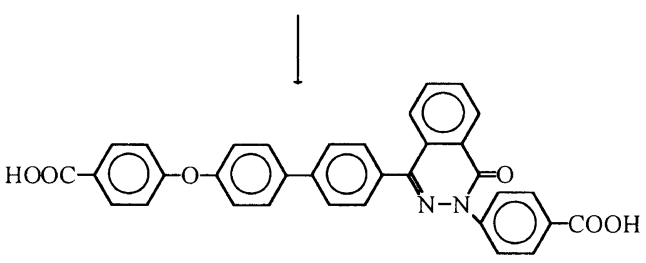

4

Scheme 1 . $8.38-8.51(\mathrm{~m}, 1 \mathrm{H}), 9.09-9.30(\mathrm{~s}, 2 \mathrm{H})$. Anal. Calcd for $\mathrm{C}_{34} \mathrm{H}_{22} \mathrm{O}_{6} \mathrm{~N}_{2}$ : C, $73.65 \% ; \mathrm{H}, 3.97 \% ; \mathrm{N}, 5.07 \%$; Found: C, $73.80 \%$; H, 3.82\%; N, $5.23 \%$.

\section{Polymer Synthesis}

Into a four-necked flask equipped with a reflux condenser, a mechanical stirrer, and a nitrogen gas inlet having a calcium chloride tube were placed $0.225 \mathrm{~g}$ of calcium chloride, and $3.75 \mathrm{ml}$ of 1-methyl-2-pyrrolidone (NMP), $2.0 \mathrm{mmol}$ of $4,2.0 \mathrm{mmol}$ of diamine, $0.9 \mathrm{ml}$ triphenyl phosphite (TPP), and $1.4 \mathrm{ml}$ of pyridine was added. The reaction mixture was heated at $110^{\circ} \mathrm{C}$ for $3 \mathrm{~h}$ with stirring. It was cooled and poured into methanol. The precipitate was collected by filtering, washed with ethanol and dried under reduced pressure.

\section{RESULTS AND DISCUSSION}

The synthesis of the diacid monomer and polymers are showed as Schemes 1 and 2 .

The high molecular weight poly(ether amide)s with inherent viscosity of $0.38-0.45$ were prepared by the biphenylphthalazinone diacid monomer and different diamines in NMP according to the phosphorylation method first reported by Yamazaki ${ }^{18}$ for the synthesis of polyamides (Scheme 2). The diamines and TPP were used as received. If purified before using, the viscosity

Table I. Properties of the polymers

\begin{tabular}{|c|c|c|c|c|}
\hline \multirow{2}{*}{ Polymer } & $\eta_{\mathrm{inh}}^{\mathrm{a}}$ & Yields & $T_{\mathrm{g}}^{\mathrm{b}}$ & $\mathrm{TGA}^{\mathrm{d}}$ \\
\hline & $\mathrm{dl} \mathrm{g} \mathrm{g}^{-1}$ & $\%$ & ${ }^{\circ} \mathrm{C}$ & ${ }^{\circ} \mathrm{C}$ \\
\hline $6 \mathbf{a}$ & 0.45 & 98 & 307 & 505 \\
\hline $\mathbf{6 b}$ & 0.37 & 96 & 252 & 500 \\
\hline $6 c$ & 0.39 & 96 & $\ldots$ & 492 \\
\hline 6d & 0.38 & 97 & 228 & 445 \\
\hline
\end{tabular}

${ }^{\text {a }}$ Measured at a concentration $0.5 \mathrm{~g} \mathrm{dL}^{-1}$ in NMP at $25^{\circ} \mathrm{C}$. ${ }^{\mathrm{b}}$ From DSC measurement conducted at a heating rate of $10^{\circ} \mathrm{Cmin}^{-1}$ in nitrogen. ${ }^{\mathrm{c}} T_{\mathrm{g}}$ was not observed in DSC trace. ${ }^{\mathrm{d}}$ Reported for $5 \%$ weight loss at a heating of $10^{\circ} \mathrm{C} \mathrm{min}^{-1}$ under $\mathrm{N}_{2}$ atmosphere.

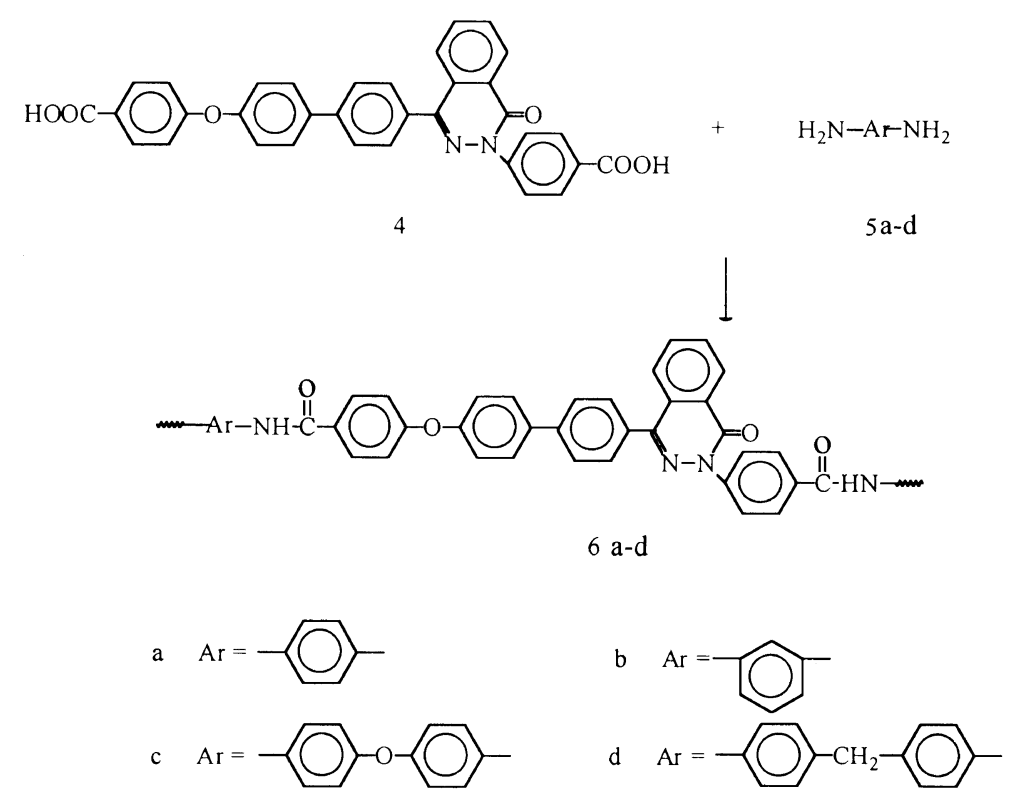

Scheme 2 . 
would have been higher. The polymers were isolated as white or yellow-white fibers and solution casting of materials from $N, N$-dimethylacetamide (DMAc) or NMP gave flexible films.

The structures of compounds $\mathbf{3}, \mathbf{4}$, and the polymers were confirmed by FT-IR and ${ }^{1} \mathrm{H}$ NMR. From FT-IR of 3, the strong carbonyl absorption around $1660 \mathrm{~cm}^{-1}$ was found indicating possibly that the compound $\mathbf{3}$ has $\mathrm{C}=\mathrm{O}$ group. It thus contains aza nitrogen arylation not its divalent phenoxide displacement. The ${ }^{1} \mathrm{H}$ NMR of 3 showed that the monomer 1 was reacted with 2 completely. The other spectrum data of $\mathbf{4}$ and $\mathbf{6}$ also agree with the proposed structures.

The solubility of these polymers was studied. These polymers have good solubility. Modification of the stiff polymer chains with naphthalene unites usually leads to an increase of solubility and phthalazinone moieties are naphthalene-like group. Thus these polymers are soluble in polar aprotic solvents such as NMP, DMAc, DMF, dimethyl sulfoxide (DMSO), etc.

From DSC measurement, glass transition temperatures were detected. The polymers showed very high $T_{\mathrm{g}} \mathrm{s}$. The $T_{\mathrm{g}} \mathrm{s}$ of $\mathbf{6 c}$ was not detected from DSC. We know that the chain rotation and interaction of the macromolecules affect the glass transition temperature. The introduction of biphenylphthalazinone moieties increases the two effects. The naphthalene-like group phthalazinone makes interactions of the macromolecules increase. So the polymers show very high $T_{\mathrm{g}} \mathrm{s}$. From the TGA of the polymers, we find these polymers have good thermal stability.

In summary, we obtained high $T_{\mathrm{g}}$ and soluble novel poly(ether amide)s containing biphenylphthalazinone moieties with high molecular weight. These polymers are soluble in organic solvents such as NMP, DMAc, DMF, DMSO, etc. The results show they are a new class of attractive thermoplastic high-performance materials. Further study of these polymers is in progress.

Acknowledgment. This work was supported by Chinese National Science Foundation.

\section{REFERENCES}

1. H. H. Yang, "Aromatic High-Strength Fibers," Wiley, New York, N.Y., 1989.

2. H. J. Jeong, M. Kakimoto, and Y. Imai, J. Polym. Sci., Part A, Polym. Chem., 29, 767 (1991).

3. F. Akutsu, H. Hirata, M. Miura, and K. Nakakubo, Makromol. Chem., Rapid. Commun., 6, 407 (1985).

4. F. Akutsu, M. Inoki, K. Sunouchi, Y. Sugama, Y, Kasashima, K. Naruchi, and M. Miura, Polymer, 39, 1637 (1998).

5. J. A. Mikroyannidis, J. Polym. Sci., Part A, Polym. Chem., 35, 1353 (1997).

6. R. A. Gaudiana, R. A. Minns, R. Sinta, N. Weeks, and H. G. Rogers, Prog. Polym. Sci., 14, 47 (1989).

7. D. Tanner, J. A. Fitzgerald, and B. R. Phillips, Angew. Chem., Adv. Mater., 101, 665 (1989).

8. Z. B. Li and M. Ueda, J. Polym. Sci., Part A, Polym Chem., 22, 3063 (1984).

9. L. H. Tagle and F. R. Diaz, Polymer, 23, 1051 (1982).

10. D. J. Liaw and K. L. Wang, J. Polym. Sci, Part A, Polym. Chem., 34, 1209 (1996).

11. K. R. Carter, P. T. Furata, and V. Gong, Macromolecules, 31 , 208 (1998).

12. F. Akutsu, T. Kataoka, K. Naruchi, M. Miura, and K. Nagakubo, Polymer, 28, 1787 (1987).

13. I. K. Spiliopoulos and J. A. Mikroyannidis, Macromolecules, 31, 1236 (1998)

14. A. A. Dembek, R. R. Burch, and A. E. Feiring, J. Am. Chem. Soc., 115, 2087 (1993).

15. Y. Z. Meng, A. S. Hay, X. G. Jian, and S. C. Tjong, J. Appl. Polym. Sci., 68, 137 (1998).

16. Y. Z. Meng, A. S. Hay, X. G. Jian, and S. C. Tjong, J. Appl. Polym. Sci., 66, 1425 (1997).

17. A. S. Hay, U.S. Patent 5237062 (1993).

18. N. Yamazaki, M. Matsumoto, and F. Higashi, J. Polym. Sci., Part A, Polym. Chem., 13, 1373 (1975). 